\title{
1 Shoot-root interaction in control of camalexin exudation in Arabidopsis
} 2

3 Anna Koprivova, Vanessa Volz, Stanislav Kopriva

\section{4}

5 Institute for Plant Sciences, Cluster of Excellence on Plant Sciences (CEPLAS),

6 University of Cologne, Zülpicher Str. 47b, 50674 Cologne, Germany

7

8

9 E-mail addresses: a.koprivova@uni-koeln.de,vvolz@smail.uni-koeln.de,

10 skopriva@uni-koeln.de

11

12 Corresponding author: Stanislav Kopriva, Phone (+49) 2214708530

13

14 Date of submission:

15 Number of figures:

16 Total word count:

17 Supplementary data:

18 Running title: Camalexin exudation

19

20 


\section{Abstract}

22 Plants exude secondary metabolites from the roots to shape the composition and

23 function of their microbiome. Many of these compounds are known for their anti24 microbial activity and are part of the plant immunity, such as the indole-derived 25 phytoalexin camalexin. Here we studied the dynamics of camalexin synthesis and 26 exudation upon induction of Arabidopsis thaliana with a plant growth promotion 27 bacteria Pseudomonas sp. CH267 or a bacterial pathogen Burkholderia glumae PG1. 28 We show that while the camalexin accumulation and exudation is more rapidly but 29 transiently induced upon interaction with the growth promoting strain, the pathogen 30 induces a higher and more stable camalexin levels. The concentration of camalexin in 31 shoots, roots and exudates is well correlated, triggering a question on the origin of the 32 exuded camalexin. By combination of experiments with cut shoots and roots and 33 grafting of wild type plant with mutants in camalexin synthesis we showed that while 34 camalexin can be produced and released by both organs, in intact plant the exuded 35 camalexin originates in the shoots. We show that camalexin synthesis in response to 36 B. glumae PG1 is dependent on cooperation of four CYP71 genes and a loss of function 37 of any of them reduces camalexin synthesis. In conclusion, camalexin synthesis seems 38 to be controlled on a whole plant level and coordinated between shoots and roots.

\section{$40 \quad$ Keywords}

41 bacterial pathogen, camalexin, grafting, plant defense, plant growth promoting

42 rhizobacteria, root exudates 


\section{INTRODUCTION}

Plants are cohabiting their natural environments with plethora of microorganisms some beneficial or commensal, some harmful (Bulgarelli et al., 2013). Plants therefore evolved number of mechanisms that enable them to communicate with the microbiota, to attract the beneficial ones and defend themselves against the harmful ones. Number of these mechanisms are based on plant metabolites that can fulfil both of these functions (reviewed in (Jacoby et al., 2020; Sasse et al., 2018). Plants produce a number of secondary compounds that are directly involved in defense (Piasecka et al., 2015). Some of these compounds are synthesised only in response to the infection, and therefore they are classified as phytoalexins, whereas others are constitutive and activated upon tissue damage or pathogen triggered signalling; these are termed phytoanticipins (VanEtten et al., 1994). Chemically, the metabolites used by plants for defense belong to all major classes of secondary compounds, terpenes, phenolic compounds, and alkaloids (Zaynab et al., 2018).

One of the best characterised classes of phytoalexins are the sulfur containing indolic compounds, such as camalexin and brassinin in the crucifers (Pedras and Yaya, 2010). Camalexin, 3-thiazol-2'-yl-indole, accumulates upon infection with fungal pathogens, such as Botrytis cinerea or Alternaria brassicicola (Bednarek et al., 2005; Kliebenstein et al., 2005; Millet et al., 2010; Thomma et al., 1999). Variation in camalexin synthesis is associated with variation to susceptibility to Botrytis in Arabidopsis accessions (Rowe and Kliebenstein, 2008). Camalexin is synthesised from tryptophan, the first step in the pathway being the production of indole-3acetaldoxime (IAOx), a common precursor for auxin, camalexin, and indole glucosinolate synthesis (Glawischnig et al., 2004). The first dedicated step in camalexin synthesis is the conversion of IAOx into indole-3-acetonitrile (IAN) by CYP71A12 and CYP71A13 (Glawischnig et al., 2004; Nafisi et al., 2007). IAN is conjugated by glutathione, which introduces the sulfur into the chemical structure and camalexin is ultimately synthesised by CYP71B15 (Geu-Flores et al., 2011; Schuhegger et al., 2006; Su et al., 2011; Zhou et al., 1999). The pathway may, however, be more complex, as two other P-450 enzymes, CYP71A27 and CYP71 A28, were associated with camalexin accumulation in roots (Koprivova et al., 2019). The role of the individual isoforms particularly in roots is thus not very clear.

In the roots, camalexin was shown to have additional function to innate immunity, as a metabolite shaping the function of root associated microbiota (Koprivova et al., 
2019). Using sulfatase activity in rhizosphere soil from Arabidopsis accessions as a measure for microbiome activity, genome wide association analysis showed that variation in CYP71A27 affects this microbial function. Loss of CYP71A27 resulted in lower sulfatase activity in soil, which could be complemented by camalexin. In addition, the cyp71A27 mutant did not benefit from plant growth promoting (PGP) effects of several rhizospheric bacteria, which again could be complemented by addition of camalexin (Koprivova et al., 2019). Camalexin is exuded from the roots (Koprivova et al., 2019; Millet et al., 2010) and may represent an important player in the mechanisms by which plants control their microbiome (Jacoby et al., 2020). However, camalexin exudation seems to be in conflict with its definition as phytoalexin, as phytoalexins act in the site of their synthesis (VanEtten et al., 1994). Thus, it is important to discover more about the nature and control of camalexin exudation.

Here we show that camalexin exudation is triggered by both pathogenic and PGP bacteria and that camalexin accumulation in exudates, roots, and leaves is highly correlated. We also reveal that both leaves and roots are able to synthesise camalexin and used grafting to show that the camalexin exuded upon treatment of roots with Burkholderia glumae originates in the shoot.

\section{RESULTS}

Both PGP and pathogenic bacteria trigger camalexin synthesis and exudation

Previous work showed that camalexin can be exuded from plant roots incubated with PGP bacteria or the bacterial-derived peptide elicitor flagellin, which is the prerequisite of camalexin function in shaping microbiome function (Koprivova et al., 2019; Millet et al., 2010). PGP bacteria and flagellin trigger also camalexin accumulation in roots, as does infection with root fungal pathogen Verticillium longisporum (Iven et al., 2012). To test, whether camalexin synthesis and exudation is triggered also by bacterial pathogens we incubated Arabidopsis growing in hydroculture with Burkholderia glumae PG1 (Gao et al., 2015) or a PGP bacterium Pseudomonas sp. CH267 (Haney et al., 2015; Koprivova et al., 2019). To obtain a better picture of a control of camalexin synthesis we analysed its accumulation in roots and shoots and in the exudates. Both bacteria triggered camalexin synthesis in all three compartments, whereas minimal camalexin levels were detected in roots and shoots of mock treated plants and no camalexin was exuded without the bacterial trigger (Figure 
112 1). The two bacteria elicited camalexin synthesis and exudation in a different way, but 113 similar in all three compartments. The PGP strain Pseudomonas sp. CH267 triggered 114 a rapid response but the accumulation of camalexin peaked between 2 and 4 days and decreased afterwards, whereas the synthesis and exudation reached a maximum after 4 to 5 days and remained high upon treatment with B. glumae PG1. In the first days, the camalexin levels were higher upon treatment with Pseudomonas sp. $\mathrm{CH} 267$ but in later stages the pathogenic strain B. glumae PG1 triggered significantly higher levels of camalexin (Figure 1). Interestingly, even though the bacteria were in contact only with the roots, the camalexin concentrations were highly correlated between shoots and roots and also between both organs and the exudates. It is thus not possible to conclude whether the exuded camalexin is synthesised in the roots or the shoots.

\section{Contribution of different isoforms of CYP71A family to camalexin synthesis}

We found previously that loss of two additional members of the CYP71A family of P450 enzymes, CYP71A27 and CYP71A28, affected camalexin levels in roots (Koprivova et al., 2019). We were therefore interested in their contribution to total camalexin synthesis and obtained all possible double and triple mutants of the four isoforms CYP71A12, CYP71A13, CYP71A27, and CYP71A28. CYP71A12 and CYP71A13 as well as CYP71A27 and CYP71A28 are two pairs of neighbouring genes, and while a double mutant cyp71a12 cyp71a13 (cyp12/13) has been produced by TALEN mutagenesis (Muller et al., 2015), double mutant of CYP71A27 and CYP71A28 is not available. These mutants were subjected to treatment with B. glumae PG1 for 3 days, leading to high synthesis of camalexin and allowing a good comparison of the individual genotypes. This analysis showed clearly that all four P450 isoforms are important for camalexin synthesis (Figure 2A). Surprisingly, loss of CYP71A27 and CYP71A28 also led to a significant reduction of camalexin synthesis in the leaves, even if the corresponding genes are not expressed there. Camalexin levels in cyp12/13 mutant is very low and in the range measured in mock treated plants, but still additional loss of either CYP71A27 or CYP71A28 lowers the camalexin accumulation further (Figure 2A). However, it needs to be seen, whether the effects of the mutations are due to loss of enzymatic activity or alteration of expression of other isoforms. Therefore, we determined the transcript levels of genes of camalexin synthesis pathway in all these mutants in roots. Inoculation with B. glumae PG1 led to increase of mRNA levels in roots of the genes for the enzymes of the canonical 
146 camalexin synthesis pathway CYP71A12, CYP71A13, and CYP7B15, as well as of

147 CYP71A27 (Figure 2B). As expected, the expression of the camalexin synthesis genes have been affected in the various mutants. The induction of CYP71A12 by B. glumae PG1 was attenuated in the single mutants of other P-450 isoforms and in the double mutant cyp13/27, but surprisingly, increased in cyp13/28 (Figure 2B). Also the induction of CYP71B15 was less pronounced in the mutants. On the other hand, CYP71A13 transcript levels were significantly elevated in genotypes with disrupted CYP71A12 already without bacterial trigger. Although the CYP71A28 mRNA was not detectable, disruption of this gene resulted in increased transcript levels of CYP71A27 both with and without inoculation (Figure 2B). Thus, the each of the four CYP71A isoforms seem to play some role in the camalexin network as loss of any of them affects at least one other member.

\section{Dissection of tissue specificity of camalexin synthesis and exudation}

The coordinated accumulation of camalexin in shoots, roots, and exudates after exposure of roots led to a question, whether the bacteria trigger camalexin synthesis also in the leaves. We, therefore, grew Arabidopsis plants on agarose plates, inoculated either the leaves or the root tips with the two bacterial strains and measured camalexin after 3 days incubation. Both inoculations triggered accumulation of camalexin in shoots and roots, but to a different extent depending on the bacterial strains. Pseudomonas sp. CH267 induced only a small camalexin accumulation, which did not differ neither in the two organs nor in the two types of inoculation and was only slightly higher than the levels found in sterile plants (Figure 3A). B. glumae PG1 triggered a similarly low camalexin synthesis when inoculated from root tip, but resulted in a large accumulation in leaves and to some extent also roots when inoculated onto leaves. Camalexin synthesis in Arabidopsis leaves thus react to B. glumae PG1 in the same way as to the fungal pathogens. The induction of camalexin synthesis in roots might be due to camalexin transport or to movement of the bacteria in the plant. We therefore used qPCR to determine bacterial titre in the plant material. No amplification was possible using primers for Pseudomonas sp. $\mathrm{CH} 267$, probably due to a low titre in our inoculations. Using primers for B. glumae PG1, however, bacteria were clearly detected in both roots and shoots, irrespective of the inoculated tissue, which reveals the mobility of this strain within the plant, both root-to-shoot and shoot-to-root

179 directions (Figure 3B). Interestingly, while in plants inoculated from the root tip the 
180 amount of camalexin approximately correlates to the bacterial titre, in the plants

181 inoculated from the leaves, the leaf camalexin concentration was almost 20-fold higher

182 in leaf than in roots, despite a similar bacterial titre (Figure 3).

183 We therefore asked, whether a communication between shoot and root affects 184 camalexin synthesis in response to B. glumae PG1. We used the hydroponics system 185 with plants growing on a nylon membrane, cut the shoots, placed them and the 186 corresponding remaining roots separately in the wells of the 12 well plates, and 187 inoculated with B. glumae PG1. Camalexin was then determined in the tissues and the exudates, as well as in shoot, root and exudates of intact plants analysed as controls (Figure 4). Both cut roots and shoots were able to exude camalexin to the solution, to levels higher than intact plants. Interestingly, whereas cut shoots accumulated more camalexin than shoots of intact plants, cut roots possessed only very low camalexin concentration compared to the intact controls. Total camalexin production in cut shoots was with $24 \pm 4 \mathrm{nmol} \mathrm{g}^{-1} \mathrm{FW}$ higher than in intact plants $\left(17 \pm 1 \mathrm{nmol} \mathrm{g}^{-1} \mathrm{FW}\right)$ and cut roots $\left(12 \pm 2 \mathrm{nmol} \mathrm{g}^{-1} \mathrm{FW}\right)$. Thus, clearly, both roots and shoots are able to synthesise camalexin and its synthesis and exudation undergoes a control dependent on root-shoot communication.

While the experiments with cut roots and shoots were informative, they do not correspond to the in vivo situation. In order to determine where the camalexin exuded by root inoculation with $B$. glumae PG1 is synthesised, we performed grafting experiments with two mutants unable to synthesis camalexin, pad3 and cyp79b2 cyp79b3 (b2/b3) (Hull et al., 2000; Zhou et al., 1999). Inoculation of roots with B. glumae PG1 resulted in accumulation of camalexin in roots, shoots and exudates of Col-0 wild type (WT) homografts but not in homografts of the two mutants (Figure 5A). Even with a large variation due to analysis of individual seedlings, it can be clearly seen that in comparison with WT homografts, similarly high camalexin accumulation was found only in heterografted shoots originating from WT. Shoots of b2/b3 mutant grafted on WT roots did not contain any camalexin higher that the background, whereas the shoots of pad3 contained a low level of camalexin. Interestingly, WT roots grafted with $b 2 / b 3$ shoots contained same level of camalexin as roots of WT homografts, while WT roots grafted with pad3 shoots did not contain any camalexin above the background. pad3 roots grafted with WT shoots accumulated camalexin but $b 2 / b 3$ roots did not (Figure 5A). Importantly, camalexin was found only 
213 on exudates of grafted plants with WT shoots. Thus, the camalexin exuded upon

214 inoculation of the roots by B. glumae PG1 must originate in the shoots.

215 When the grafted plants were inoculated with B. glumae PG1 on the leaves, camalexin 216 was found mainly in shoots of WT homografts with much lower levels in roots and 217 concentration not different to background in the exudates (Figure 5B). In the 218 heterografts with $b 2 / b 3$, only the tissues originating from WT accumulated camalexin, 219 and none was exuded, whereas in grafts with pad3 high level of camalexin was found 220 in the shoots originating from WT, and low levels in both types of roots. Thus in whole 221 plants camalexin synthesis seems to be tightly controlled and primarily occurring in 222 the shoots.

\section{DISCUSSION}

225 Camalexin is a relatively new addition to the list of plant metabolites that shape the 226 root associated microbiome (Jacoby et al., 2020; Koprivova et al., 2019). Similar to 227 other such compounds, e.g. coumarins or benzoxazinoids (de Bruijn et al., 2018; 228 Stringlis et al., 2018), camalexin has first been characterised for its antimicrobial properties (Rogers et al., 1996). However, in the rhizosphere these compounds affect the microbiota in a way that they support plant fitness and performance. For example, coumarins exuded from plants were shown to affect the communities to improve plant iron nutrition (Harbort et al., 2020). Camalexin was shown to affect the microbial sulfatase activity in rhizosphere soil that mineralises organic sulfur and so the bacteria help plants to access this sulfur pool (Kertesz and Mirleau, 2004; Koprivova et al., 2019). Thus, camalexin has to be exuded to fulfil this function, however, unlike the coumarins or benzoxazinoids, camalexin has not been found in roots exudates unless elicited (Millet et al., 2010; Monchgesang et al., 2016; Neal et al., 2012). In addition, while the other metabolites characterised so far change the taxonomic assembly of the microbial community, this still needs to be tested for camalexin.

240 Since camalexin has always been a prime example of a phytoalexin, i.e. acting locally

241 at the site of pathogen attack, it is not obvious how camalexin exudation from the roots 242 is regulated. Previous work focused on camalexin synthesis in the leaves as a reaction to leaf pathogens (Glazebrook and Ausubel, 1994; Kliebenstein et al., 2005; Thomma et al., 1999). It was shown previously that the exudation could be elicited by flagellin or by PGP bacteria (Koprivova et al., 2019; Millet et al., 2010), but to relatively low levels. It was therefore important that high camalexin exudation can be triggered by 
247 the pathogenic bacteria B. glumae PG1 as a robust high exudation is needed to dissect

248 the regulation. Interestingly, the dynamics of camalexin synthesis and exudation

249 responds differentially to pathogenic and commensual or beneficial bacteria. While

250 the PGP strain seemed to trigger the camalexin synthesis quicker, the response was

251 only transient. The pathogenic bacterial strain seemed to be slower in initiation of the

252 synthesis, but this was stronger and remained active for longer and did not diminish.

253 The levels of camalexin in roots of B. glumae PG1 treated Arabidopsis plants in our

254 system were similar to those in roots treated with $V$. longisporum (Iven et al., 2012).

255 It seems therefore, that while the camalexin synthesis is initiated by both types of

256 microorganisms, only upon interaction with pathogens the synthesis and exudation is

257 sustained long-term. Similarly, when the plants were grown on agar plates and

258 inoculated with from the leaves, B. glumae PG1 induced much higher camalexin levels

259 than Pseudomonas sp. CH267 (Figure 3). B. glumae PG1 can thus be used as a tool to

260 study the control of camalexin synthesis and exudation.

261 The first question addressed using B. glumae PG1 was the contribution of the "old"

262 (CYP71A12 and A13) and "new" (A27 and A28) CYP71A isoforms to camalexin

263 synthesis and exudation. All mutants clearly showed reduced total accumulation of

264 camalexin, when the concentrations in shoots, roots, and exudates were summed

265 (Figure 2A). This was particularly true for the concentration in the roots, which

266 contributed most to the total camalexin; not surprisingly, as the pathogen was

267 inoculated by the roots. However, with exception of cyp71a28 all other mutants

268 showed also reduced accumulation in the shoots. This result is in contrast to previous

269 experiments with the "old" cyp71a12 and cyp71a13, as in the former, upon abiotic

270 elicitation with UV light or $\mathrm{AgNO}_{3}$ camalexin in leaves was not affected and upon

271 treatment with spores of fungal pathogen Plectosphaerella cucumerina BMM even

272 increased (Muller et al., 2015; Pastorczyk et al., 2020). On the other hand it agrees

273 with the measurements of camalexin in roots of soil grown plants, where all four single

274 mutants showed lower concentrations (Koprivova et al., 2019). The data also clearly

275 demonstrate the very high contribution of CYP71A12 and CYP71A13 to camalexin

276 synthesis in all compartments, despite previous conclusion that CYP71A12 is

277 responsible for root synthesis and exudation upon elicitation with flagellin (Millet et

278 al., 2010). However, the data also show that even in the absence of these two enzymes,

279 some camalexin is produced and this production is dependent on CYP71A27.

280 Interestingly, the loss of the individual genes affects also transcript levels of the other 
281 members of the biosynthesis network (Figure 2B). With one notable exception, an induction of CYP71A13 in the cyp71a12 background, the level of induction of the genes by $B$. glumae PG1 was attenuated in the mutants. Since the enzymes of camalexin synthesis form a metabolon (Mucha et al., 2019) this might be a mechanism to prevent accumulation of proteins that cannot be part of this structure.

The analysis of camalexin in the 3 compartments of different mutants showed that its concentrations correlate well between all three of them. The genes for camalexin synthesis are also expressed in both shoots and roots. Thus, the camalexin in the exudates might originate in the roots as well as in the leaves.

To find out which organ is responsible for the synthesis of camalexin found in exudates we designed two experiments. In a simple approach we cut shoots and roots and incubated them separately with the bacterial pathogen (Figure 4). This experiment revealed that both shoots and roots are autonomous in camalexin synthesis. Shoots alone even produced more camalexin than the whole plants, that can be explained by the direct leakage of the synthesised camalexin and also by a more rapid contact of the shoots with the bacteria. The inoculation of leaves with B. glumae PG1 (Figure 3) resulted in a much higher camalexin accumulation in leaves compared to the hydroculture setup (Figures 1 and 2), which is consistent with the high camalexin in cut shoots. The high camalexin production in the cut roots, on the other hand, was unexpected, since in previous experiments with inoculation from the leaves the same bacterial titre that triggered accumulation of ca. $40 \mathrm{nmol} \mathrm{mg}^{-1} \mathrm{FW}$ in leaves induced only $1 \mathrm{nmol} \mathrm{mg}^{-1} \mathrm{FW}$ in the roots. This means that a coordination between shoots and roots is necessary to prevent camalexin overproduction. There are number of examples how roots and shoots communicate in defense, from the resistance against leaf pathogens induced by the rhizobacterium Pseudomonas fluorescens SS101, which also involves camalexin (van de Mortel et al., 2012), to the coordination in jasmonate signalling for resistance to nematodes (Wang et al., 2019). Camalexin synthesis can be affected by auxin and by miRNA393, both known long-distance signals (Robert-

311 The existence of such coordination was clearly demonstrated in the second approach, 312 using grafting with mutants that do not synthesise camalexin, pad3 and cyp79b2 $313 c y p 79 b 3$. Any camalexin found in the grafted tissues originating from the mutants must 314 be transported from WT and thus evidence for a long-distance transport. Indeed, the 
315 grafting experiment showed unequivocally that camalexin exuded from the roots originates in the shoots. Camalexin was found in amounts over background only in exudates from heterografts with WT shoots, but not roots. Interestingly, when the plants were inoculated onto the leaves, camalexin synthesis in the leaves was induced to the same degree, but none of this camalexin was exuded. Thus the plants seem to recognise where the infection originates and steer the camalexin synthesis there. This process requires a sophisticated coordination between the roots and the shoots, and cannot rely solely on the actual perception of the bacteria, as seen also in Figure 3, where the same bacterial titres triggered different camalexin levels in shoots and roots. It remains to be seen whether camalexin exudation in response to PGP bacteria undergoes the same whole plant regulation or whether the camalexin is produced locally, in the root, as might be indicated by the lower production and different dynamics.

328 In conclusion, here we show that inoculation of Arabidopsis root with a bacterial pathogen B. glumae PG1 triggers camalexin synthesis in shoots and roots and its exudation. The camalexin can be produced and released by both organs, but in intact plant the exuded camalexin originates in the shoots. We show that the camalexin synthesis genes are tightly regulated and loss of function of any of them affects total camalexin synthesis. Finally, we conclude that camalexin synthesis is controlled by a whole plant regulation with a need for shoot root communication.

\section{MATERIALS AND METHODS}

\section{Plant material and growth conditions}

Arabidopsis thaliana L. accession Col-0 was used as wild type alongside mutants in camalexin synthesis cyp71a12 (GABI_127H03), cyp71a13 (SALK_105136), pad3 (SALK_026585), cyp71a27 (SALK_053817) and cyp71a28 (SALK_ 064792). The double mutants cyp71a12 cyp71a13 and cyp79b2 cyp79b3 were obtained from $\mathrm{H}$.

342 Frerigmann and T. Gigolashvili, University of Cologne, respectively.

343 For camalexin and expression analyses plants were surface sterilized with chlorine gas.

344 Seeds were suspended in $0.1 \%$ agarose, distributed onto square $1 \mathrm{~cm} \mathrm{x} 1 \mathrm{~cm}$ sterile nylon membranes (about 30 seeds per sample) and placed in 12 well plates on top of $1 \mathrm{ml}$ of $1 / 2$ Murashige Skoog (MS) medium with $0.5 \%$ sucrose. After stratification for

3472 days in dark and cold the plates were transferred to $22^{\circ} \mathrm{C}$ and kept in dark for 3 days 348 to promote etiolation, which greatly simplifies the separation of shoots from 
membranes. Afterwards the plates were incubated at long day conditons (16 h light/ 8 h dark), $120 \mu \mathrm{E} \mathrm{m}^{-2} \mathrm{~S}^{-1}$, and at $22^{\circ} \mathrm{C}$ for further 7 days. The medium was then replaced with $1 / 2$ MS without sucrose and the plants incubated for 24 hours before inoculation with the bacteria or mock and incubated further in the same conditions for 3 days, unless specified otherwise.

For the experiments with cut shoots and roots immediately before inoculation the shoots were cut with scissors and roots were freed from the membrane and placed directly into the nutrient solution.

\section{Bacterial strains and conditions for cocultivation experiments}

For co cultivation experiments 2 bacterial strains were used, Pseudomonas sp. CH267 (Haney et al., 2015), obtained from J. R. Dinneny, Stanford University and B. glumae PG1 (Gao et al., 2015), obtained from K.-E. Jäger, Heinrich Heine Universität Düsseldorf, Germany. The bacteria were kept as glycerol stocks and plated freshly before experiment on LB plates supplemented with appropriate antibiotics.

For inoculation, overnight bacterial cultures were washed two times with sterile 10

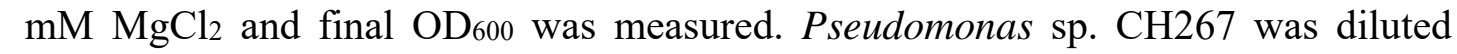
stepwise to $\mathrm{OD}_{600}=0.0001$, and B. glumae PG1 to $\mathrm{OD}_{600}=0.0005$ in $10 \mathrm{mM} \mathrm{MgCl}_{2}$. Eight $\mu 1$ of these suspensions were used for inoculation into each well. Eight $\mu 1$ of 10 $\mathrm{mM} \mathrm{MgCl}_{2}$ was used as mock treatment. Samples for DNA, RNA and camalexin (shoots, roots and exudates) were harvested after 3 days of inoculation, except the time course experiments.

Alternatively, plants were grown on square Petri dishes with $1 / 2$ MS with sucrose for 18 days and inoculated with $8 \mu 1$ of suspensions of Pseudomonas sp. $\mathrm{CH} 267$ (OD $600=$ $0.0001)$ or B. glumae PG1 $\left(\mathrm{OD}_{600}=0.0005\right)$ onto leaves or the bottom $2 \mathrm{~mm}$ of root tips. After 30 min drying the plates were returned to growth cabinet and grown for 3 days at long days.

\section{Camalexin measurements}

Camalexin was extracted from 5-30 mg of plant material as described in (Koprivova et al., 2019). For extraction of camalexin from exudates the media were centrifuged at maximum speed for $20 \mathrm{~min}$ at $18^{\circ} \mathrm{C}$ and purified using $1 \mathrm{ml}$ solid phase extraction tubes (Discovery- DSC18) according to manufacturer's instructions. Samples were eluted with $90 \%$ (V/V) acetonitrile and $0.1 \%$ (V/V) formic acid, dried in a speed vac 
and dissolved in $50 \mu \mathrm{l}$ of DMSO. $20 \mu \mathrm{l}$ was injected into HPLC and analysed as described above. For the quantification external standards were used ranging from 1 pg to $1 \mathrm{ng}$ per $\mu 1$.

\section{Grafting experiments}

For grafting, the selected genotypes were first grown on vertical Petri dishes containing $1 / 2$ MS with sucrose and $0.8 \%$ agarose for 5-6 days at short day conditions (10 h light $/ 14 \mathrm{~h}$ dark) and $22^{\circ} \mathrm{C}$. Grafting was performed under binocular microscope in sterile conditions and the grafts were transferred onto fresh plates for further 18 days under the same short day light conditions. Graft unions were examined under the binocular to identify adventitious root formation. Healthy grafts were carefully transferred into 12 well plates with $1 \mathrm{ml}$ of $1 / 2 \mathrm{MS}$ medium placing the shoots onto sterile cut cups from $0.5 \mathrm{ml}$ Eppendorf tubes in order to prevent them from direct contact with the liquid. The plants were inoculated with $8 \mu 1$ of B. glumae PG1 suspension at $\mathrm{OD}_{600}=0.0005$ into the solution or onto the leaves and further incubated for 3 days. Camalexin analysis in shoots, roots, and exudates of individual plants was performed as described above.

\section{Expression analysis}

To determine transcript levels total RNA was isolated by standard phenol/chlorophorm extraction and $\mathrm{LiCl}$ precipitation. First strand cDNA synthesis was performed using QuantiTect Reverse transcription Kit (Quiagen) from $800 \mathrm{ng}$ of total RNA. Quantitative real time RT-PCR (qPCR) was performed using gene-specific primers (Supplemental Table 1) and the fluorescent dye SYBR Green (Promega). All quantifications were normalized to the TIP41 (AT4G34270) gene. The RT-PCR reactions were performed in duplicate for each of the 4 independent samples.

\section{Determination of bacterial titre}

For the estimation of bacterial titre using qPCR the method from (Ross and Somssich, 2016) was adapted. Genomic DNA was extracted using buffer containing 0.025 M EDTA, 0.2 M Tris pH 8.0, 0.25 M NaCl and 0.5\% SDS. After 10 min incubation at $65^{\circ} \mathrm{C}$ and subsequent centrifugation, supernatant was precipitated with equal volume of isopropanol, washed with $70 \%$ ethanol and resuspended in $100 \mu l$ of sterile water. For the qPCR 13 ng of corresponding DNA samples were used with Arabidopsis (At 
417 primer AT4G26410) and B. glumae PG1 specific primer (Burk1 for NR042931). The

418 qPCR conditions were the same as for expression analysis. The qPCR reactions were

419 performed in duplicate for each of the 4 independent samples. To relate the qPCR

420 results to the bacterial titre, first serial dilutions of bacterial suspensions of different

421 OD 600 have been plated on LB plates and the colonies were counted manually to link

$422 \mathrm{OD}_{600}$ and cfu. Subsequently, $10 \mu \mathrm{l}$ of five 10-fold dilutions of bacterial suspensions

423 with initial $\mathrm{OD}_{600}=1.8$ were added to $30 \mathrm{mg}$ of Arabidopsis leaves and the DNA

424 extracted and analysed as above. Using calibration curves plotting $\Delta \mathrm{Ct}\left(\mathrm{Ct}_{\mathrm{Bg}}-\mathrm{Ct}_{\mathrm{At}}\right)$

425 and the cfu against the $\log _{10} \mathrm{OD}_{600}$ the bacterial titre can be estimated from the $\Delta \mathrm{Ct}$

426 values.

\section{ACKNOWLEDGEMENTS}

430 Research in SK's lab is funded by the Deutsche Forschungsgemeinschaft (DFG) under

431 Germany's Excellence Strategy - EXC 2048/1 - project 390686111 and within the 432 SPP 2125 DECRyPT.

433

434 


\section{FIGURE LEGENDS}

Figure 1. Camalexin accumulation upon inoculation with PGP or pathogen bacteria.

Arabidopsis plants were grown on a nylon net in hydroculture for 10 days and inoculated in the solution with Pseudomonas sp. CH267, B. glumae PG1 (BG), or $\mathrm{MgCl}_{2}$ as mock. Camalexin was measured in leaves (A), roots (B), and exudates (C) sampled daily over 6 days. Data are presented as means \pm S.D. from 4 biological replicates, each corresponding to at least 30 seedlings. Asterisks mark significant differences between the values of $\mathrm{CH} 267$ and PG1 treated plants $(\mathrm{P}<0.05, \mathrm{~T}$-test $)$.

Figure 2. Characterisation of mutants in CYP71A genes involved in camalexin synthesis.

The seedlings were grown on a nylon net in hydroculture for 10 days, inoculated in the solution with $B$. glumae $\mathrm{PG1}$ or $\mathrm{MgCl}_{2}$ as mock and incubated for 3 days. $\mathbf{A}$ Camalexin was measured in leaves, roots, and exudates of B. glumae PG1 treated plants. Data are presented as means \pm S.D. from 4 biological replicates, each corresponding to at least 30 seedlings. Different letters mark significant differences in total camalexin (shoots + roots + exudates) between the genotypes $(\mathrm{P}<0.05$, ANOVA). B Transcript levels of the genes of camalexin synthesis were compared by RT-qPCR in roots of mock $(\mathrm{M})$ and B. glumae PG1 $(\mathrm{BG})$ treated plants. Data are shown as heatmap of relative expression.

\section{Figure 3. Tissue specificity of camalexin synthesis}

Arabidopsis seedlings were grown for 14 days on an agar plate, inoculated with Pseudomonas sp. CH267 (A) or B. glumae PG1 (BG) (B) either on the leaves or on the root tips, and incubated for 3 days. Camalexin accumulation in leaves and roots was determined by HPLC. Data are presented as means \pm S.D. from 3 biological replicates, each corresponding to 3 individual roots or shoots. C DNA was isolated from the roots and shoots and subjected to qPCR with primers against B. glumae PG1 and Arabidopsis TIP41 gene as control. Using previously established calibration between $\mathrm{Ct}$ values, OD and cfu, the qPCR data were expressed as cfu, presented as means \pm S.D. from 4 biological replicates, each corresponding to 3 individual roots or shoots. Different letters mark values significantly different at $\mathrm{P}<0.05$ (T-test).

\section{Figure 4. Camalexin in cut roots and shoots}

Arabidopsis seedlings were grown on a nylon net in hydroculture for 10 days, the shoots were cut with scissors, the roots removed from the net and both placed 
470 separately to the solution. The shoots, roots, and intact plants were inoculated with $B$.

471 glumae PG1 and further incubated for 3 days. Camalexin accumulation in shoots,

472 roots, and exudates was determined by HPLC. Data are presented as box plots from at

473 least 8 biological replicates, each corresponding to about 30 individual roots or shoots.

474 The box extends from the $25^{\text {th }}$ to $75^{\text {th }}$ percentiles, the line is plotted at the median, the

475 whiskers extend from minimum to maximum values.

\section{Figure 5. Analysis of camalexin in grafted plants.}

477 Homografts of Arabidopsis WT, cyp79b2 cyp79b3 (b2/b3), and pad3 and the

478 heterografts of the WT with the mutants were grown for 18 after the grafting,

479 transferred onto cut caps of Eppendorf tubes and placed with only the roots submerged

480 into the hydroculture solution. The plants were then inoculated with B. glumae PG1

481 into the solution (A) or onto the leaves (B) and further incubated for 3 days. Camalexin

482 accumulation in shoots, roots, and exudates was determined by HPLC. Data are

483 presented as box plots from at least 8 individual grafts. The box extends from the $25^{\text {th }}$

484 to $75^{\text {th }}$ percentiles, the line is plotted at the median, the whiskers extend from minimum

485 to maximum values. 
488

\section{REFERENCES}

Bednarek P, Schneider B, Svatos A, Oldham NJ, Hahlbrock K. 2005. Structural complexity, differential response to infection, and tissue specificity of indolic and phenylpropanoid secondary metabolism in Arabidopsis roots. Plant Physiology 138, 1058-1070.

\section{Bulgarelli D, Schlaeppi K, Spaepen S, Ver Loren van Themaat E, Schulze-Lefert} P. 2013. Structure and functions of the bacterial microbiota of plants. Annual Review of Plant Biology, Vol 62 64, 807-838.

de Bruijn WJC, Gruppen H, Vincken JP. 2018. Structure and biosynthesis of benzoxazinoids: Plant defence metabolites with potential as antimicrobial scaffolds. Phytochemistry 155, 233-243.

Gao R, Krysciak D, Petersen K, Utpatel C, Knapp A, Schmeisser C, Daniel R, Voget S, Jaeger KE, Streit WR. 2015. Genome-wide RNA sequencing analysis of quorum sensing-controlled regulons in the plant-associated Burkholderia glumae PG1 strain. Appl Environ Microbiol 81, 7993-8007.

Geu-Flores F, Moldrup ME, Bottcher C, Olsen CE, Scheel D, Halkier BA. 2011. Cytosolic gamma-glutamyl peptidases process glutathione conjugates in the biosynthesis of glucosinolates and camalexin in Arabidopsis. Plant Cell 23, 24562469.

Glawischnig E, Hansen BG, Olsen CE, Halkier BA. 2004. Camalexin is synthesized from indole-3-acetaldoxime, a key branching point between primary and secondary metabolism in Arabidopsis. Proc Natl Acad Sci U S A 101, 8245-8250.

Glazebrook J, Ausubel FM. 1994. Isolation of phytoalexin-deficient mutants of Arabidopsis thaliana and characterization of their interactions with bacterial pathogens. Proc Natl Acad Sci U S A 91, 8955-8959.

Haney CH, Samuel BS, Bush J, Ausubel FM. 2015. Associations with rhizosphere bacteria can confer an adaptive advantage to plants. Nat Plants 1, 15051.

Harbort CJ, Hashimoto M, Inoue H, Niu Y, Guan R, Rombola AD, Kopriva S, Voges M, Sattely ES, Garrido-Oter R, Schulze-Lefert P. 2020. Root-Secreted Coumarins and the Microbiota Interact to Improve Iron Nutrition in Arabidopsis. Cell Host Microbe 28, 825-837 e826. 
519 Hull AK, Vij R, Celenza JL. 2000. Arabidopsis cytochrome P450s that catalyze the

520 first step of tryptophan-dependent indole-3-acetic acid biosynthesis. Proc Natl Acad 521 Sci U S A 97, 2379-2384.

522 Iven T, Konig S, Singh S, Braus-Stromeyer SA, Bischoff M, Tietze LF, Braus GH,

523 Lipka V, Feussner I, Droge-Laser W. 2012. Transcriptional activation and 524 production of tryptophan-derived secondary metabolites in arabidopsis roots 525 contributes to the defense against the fungal vascular pathogen Verticillium 526 longisporum. Molecular Plant 5, 1389-1402.

527 Jacoby RP, Koprivova A, Kopriva S. 2020. Pinpointing secondary metabolites that 528 shape the composition and function of the plant microbiome. Journal of Experimental 529 Botany.

530 Kertesz MA, Mirleau P. 2004. The role of soil microbes in plant sulphur nutrition. Journal of Experimental Botany 55, 1939-1945.

532 Kliebenstein DJ, Rowe HC, Denby KJ. 2005. Secondary metabolites influence 533 Arabidopsis/Botrytis interactions: variation in host production and pathogen 534 sensitivity. Plant Journal 44, 25-36.

535 Koprivova A, Schuck S, Jacoby RP, Klinkhammer I, Welter B, Leson L, Martyn 536 A, Nauen J, Grabenhorst N, Mandelkow JF, Zuccaro A, Zeier J, Kopriva S. 2019. 537 Root-specific camalexin biosynthesis controls the plant growth-promoting effects of 538 multiple bacterial strains. Proc Natl Acad Sci U S A 116, 15735-15744.

539 Millet YA, Danna CH, Clay NK, Songnuan W, Simon MD, Werck-Reichhart D, 540 Ausubel FM. 2010. Innate immune responses activated in Arabidopsis roots by 541 microbe-associated molecular patterns. Plant Cell 22, 973-990.

542 Monchgesang S, Strehmel N, Schmidt S, Westphal L, Taruttis F, Muller E, 543 Herklotz S, Neumann S, Scheel D. 2016. Natural variation of root exudates in 544 Arabidopsis thaliana-linking metabolomic and genomic data. Sci Rep 6, 29033.

545 Mucha S, Heinzlmeir S, Kriechbaumer V, Strickland B, Kirchhelle C, 546 Choudhary M, Kowalski N, Eichmann R, Huckelhoven R, Grill E, Kuster B, 547 Glawischnig E. 2019. The Formation of a Camalexin Biosynthetic Metabolon. Plant 548 Cell 31, 2697-2710.

549 Muller TM, Bottcher C, Morbitzer R, Gotz CC, Lehmann J, Lahaye T, 550 Glawischnig E. 2015. TRANSCRIPTION ACTIVATOR-LIKE EFFECTOR 
551 NUCLEASE-Mediated Generation and Metabolic Analysis of Camalexin-Deficient

552 cyp71a12 cyp71a13 Double Knockout Lines. Plant Physiology 168, 849-858.

553 Nafisi M, Goregaoker S, Botanga CJ, Glawischnig E, Olsen CE, Halkier BA, 554 Glazebrook J. 2007. Arabidopsis cytochrome P450 monooxygenase 71A13 catalyzes

555 the conversion of indole-3-acetaldoxime in camalexin synthesis. Plant Cell 19, 20395562052.

557 Neal AL, Ahmad S, Gordon-Weeks R, Ton J. 2012. Benzoxazinoids in root 558 exudates of maize attract Pseudomonas putida to the rhizosphere. PLoS One 7, e35498.

559 Pastorczyk M, Kosaka A, Pislewska-Bednarek M, Lopez G, Frerigmann H, 560 Kulak K, Glawischnig E, Molina A, Takano Y, Bednarek P. 2020. The role of 561 CYP71A12 monooxygenase in pathogen-triggered tryptophan metabolism and 562 Arabidopsis immunity. New Phytologist 225, 400-412.

563 Pedras MS, Yaya EE. 2010. Phytoalexins from Brassicaceae: news from the front. 564 Phytochemistry 71, 1191-1197.

565 Piasecka A, Jedrzejczak-Rey N, Bednarek P. 2015. Secondary metabolites in plant 566 innate immunity: conserved function of divergent chemicals. New Phytologist 206, 567 948-964.

568 Robert-Seilaniantz A, MacLean D, Jikumaru Y, Hill L, Yamaguchi S, Kamiya Y, 569 Jones JD. 2011. The microRNA miR393 re-directs secondary metabolite biosynthesis 570 away from camalexin and towards glucosinolates. Plant Journal 67, 218-231.

571 Rogers EE, Glazebrook J, Ausubel FM. 1996. Mode of action of the Arabidopsis 572 thaliana phytoalexin camalexin and its role in Arabidopsis-pathogen interactions. Mol 573 Plant Microbe Interact 9, 748-757.

574 Ross A, Somssich IE. 2016. A DNA-based real-time PCR assay for robust growth 575 quantification of the bacterial pathogen Pseudomonas syringae on Arabidopsis 576 thaliana. Plant Methods 12, 48.

577 Rowe HC, Kliebenstein DJ. 2008. Complex genetics control natural variation in 578 Arabidopsis thaliana resistance to Botrytis cinerea. Genetics 180, 2237-2250.

579 Sasse J, Martinoia E, Northen T. 2018. Feed Your Friends: Do Plant Exudates Shape 580 the Root Microbiome? Trends in Plant Science 23, 25-41. 
581 Schuhegger R, Nafisi M, Mansourova M, Petersen BL, Olsen CE, Svatos A, 582 Halkier BA, Glawischnig E. 2006. CYP71B15 (PAD3) catalyzes the final step in 583 camalexin biosynthesis. Plant Physiology 141, 1248-1254.

584 Stringlis IA, Yu K, Feussner K, de Jonge R, Van Bentum S, Van Verk MC, 585 Berendsen RL, Bakker P, Feussner I, Pieterse CMJ. 2018. MYB72-dependent 586 coumarin exudation shapes root microbiome assembly to promote plant health. Proc 587 Natl Acad Sci U S A 115, E5213-E5222.

588 Su T, Xu J, Li Y, Lei L, Zhao L, Yang H, Feng J, Liu G, Ren D. 2011. Glutathione589 indole-3-acetonitrile is required for camalexin biosynthesis in Arabidopsis thaliana. $590 \quad$ Plant Cell 23, 364-380.

591 Thomma BP, Nelissen I, Eggermont K, Broekaert WF. 1999. Deficiency in 592 phytoalexin production causes enhanced susceptibility of Arabidopsis thaliana to the 593 fungus Alternaria brassicicola. Plant Journal 19, 163-171.

594 van de Mortel JE, de Vos RC, Dekkers E, Pineda A, Guillod L, Bouwmeester K, 595 van Loon JJ, Dicke M, Raaijmakers JM. 2012. Metabolic and transcriptomic 596 changes induced in Arabidopsis by the rhizobacterium Pseudomonas fluorescens 597 SS101. Plant Physiology 160, 2173-2188.

598 VanEtten HD, Mansfield JW, Bailey JA, Farmer EE. 1994. Two Classes of Plant 599 Antibiotics: Phytoalexins versus "Phytoanticipins". Plant Cell 6, 1191-1192.

600 Wang G, Hu C, Zhou J, Liu Y, Cai J, Pan C, Wang Y, Wu X, Shi K, Xia X, Zhou 601 Y, Foyer CH, Yu J. 2019. Systemic Root-Shoot Signaling Drives Jasmonate-Based 602 Root Defense against Nematodes. Curr Biol 29, 3430-3438 e3434.

603 Zaynab M, Fatima M, Abbas S, Sharif Y, Umair M, Zafar MH, Bahadar K. 2018. 604 Role of secondary metabolites in plant defense against pathogens. Microb Pathog 124, $605 \quad 198-202$.

606 Zhou N, Tootle TL, Glazebrook J. 1999. Arabidopsis PAD3, a gene required for 607 camalexin biosynthesis, encodes a putative cytochrome P450 monooxygenase. Plant 608 Cell 11, 2419-2428. 


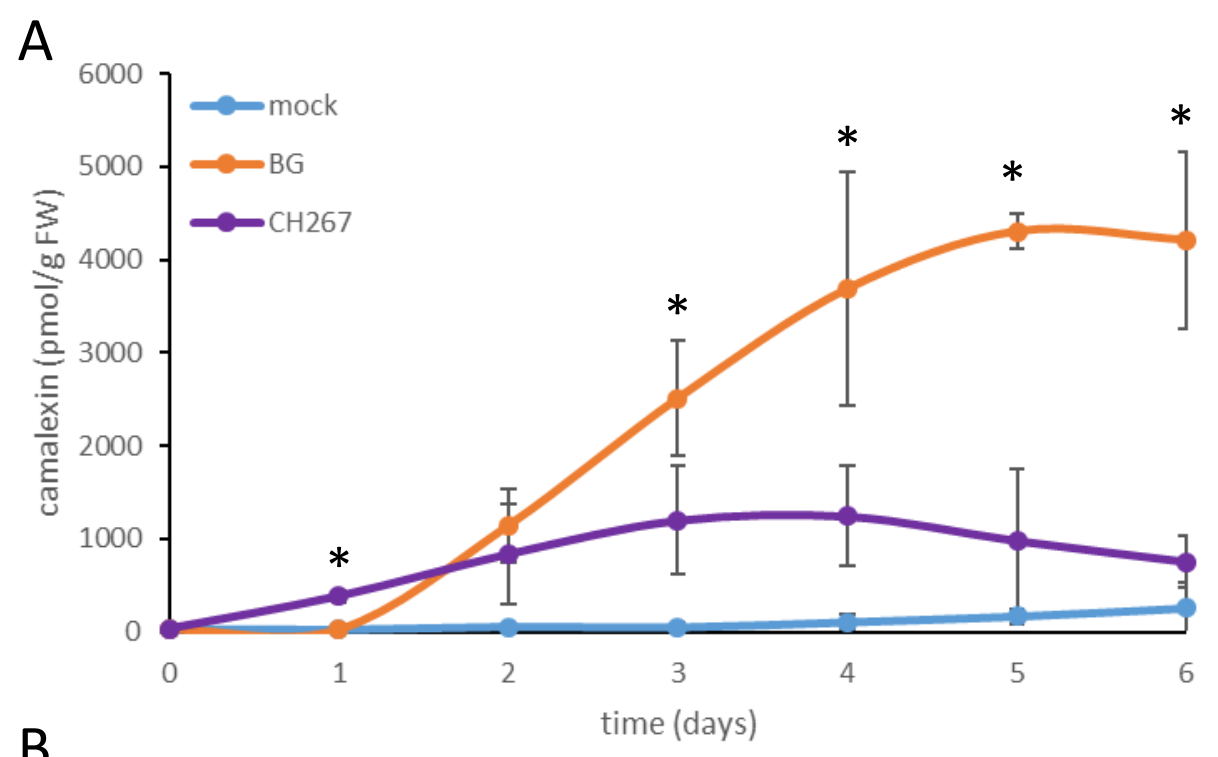

B
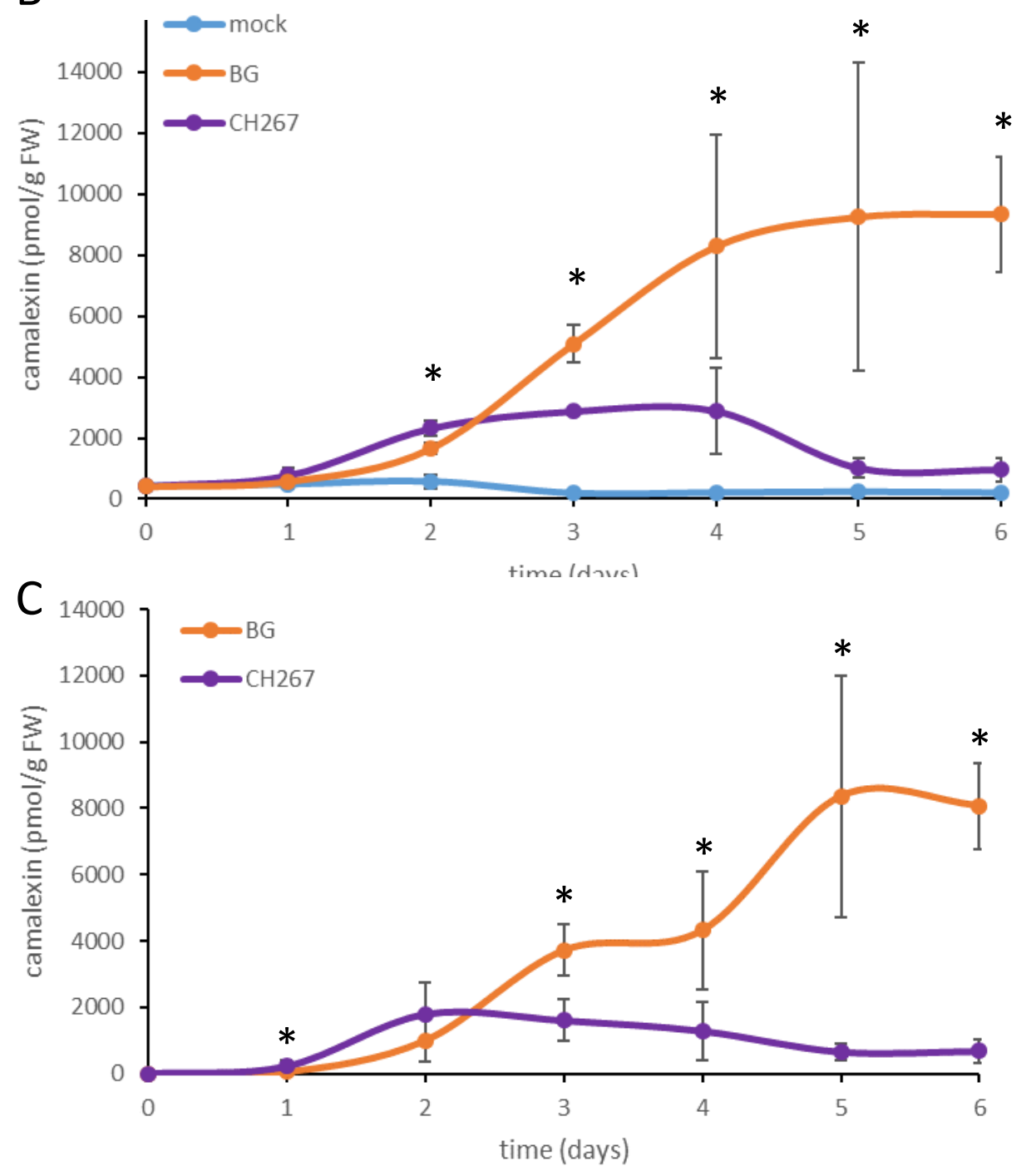

Figure 1. Camalexin accumulation upon inoculation with PGP or pathogen bacteria.

Arabidopsis plants were grown on a nylon net in hydroculture for 10 days and inoculated in the solution with Pseudomonas sp. CH267, B. glumae PG1 (BG), or $\mathrm{MgCl}_{2}$ as mock. Camalexin was measured in leaves (A), roots $(\mathbf{B})$, and exudates $(\mathbf{C})$ sampled daily over 6 days. Data are presented as means \pm S.D. from 4 biological replicates, each corresponding to at least 30 seedlings. Asterisks mark significant differences between the values of $\mathrm{CH} 267$ and $\mathrm{PG} 1$ treated plants $(\mathrm{P}<0.05$, T-test $)$. 

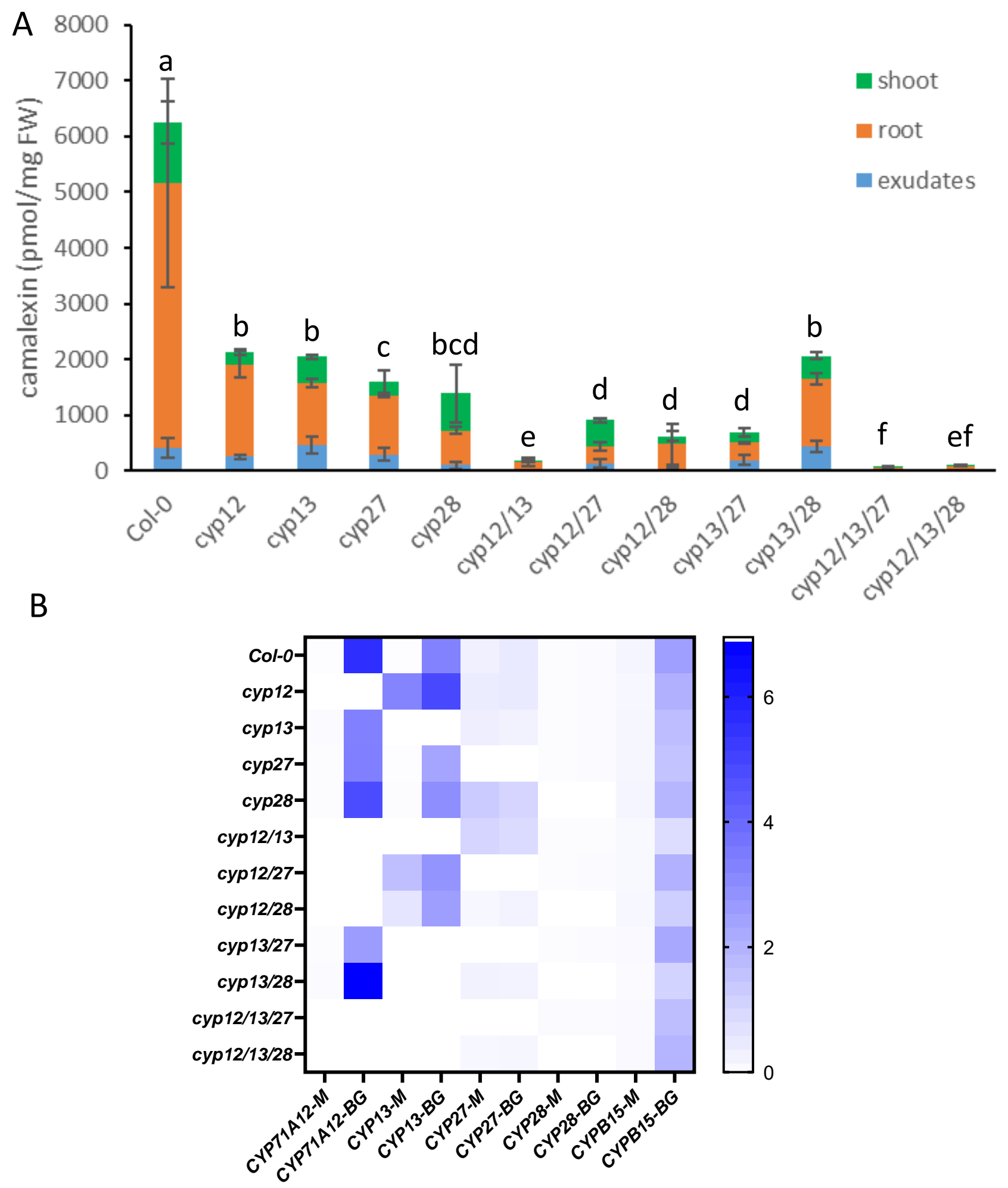

Figure 2. Characterisation of mutants in CYP71A genes involved in camalexin synthesis.

The seedlings were grown on a nylon net in hydroculture for 10 days, inoculated in the solution with $B$. glumae PG1 or $\mathrm{MgCl}_{2}$ as mock and incubated for 3 days. A Camalexin was measured in leaves, roots, and exudates of B. glumae PG1 treated plants. Data are presented as means \pm S.D. from 4 biological replicates, each corresponding to at least 30 seedlings. Different letters mark significant differences in total camalexin (shoots + roots + exudates) between the genotypes ( $\mathrm{P}<0.05$, ANOVA). B Transcript levels of the genes of camalexin synthesis were compared by RT-qPCR in roots of mock (M) and B. glumae PG1 (BG) treated plants. Data are shown as heatmap of relative expression. 

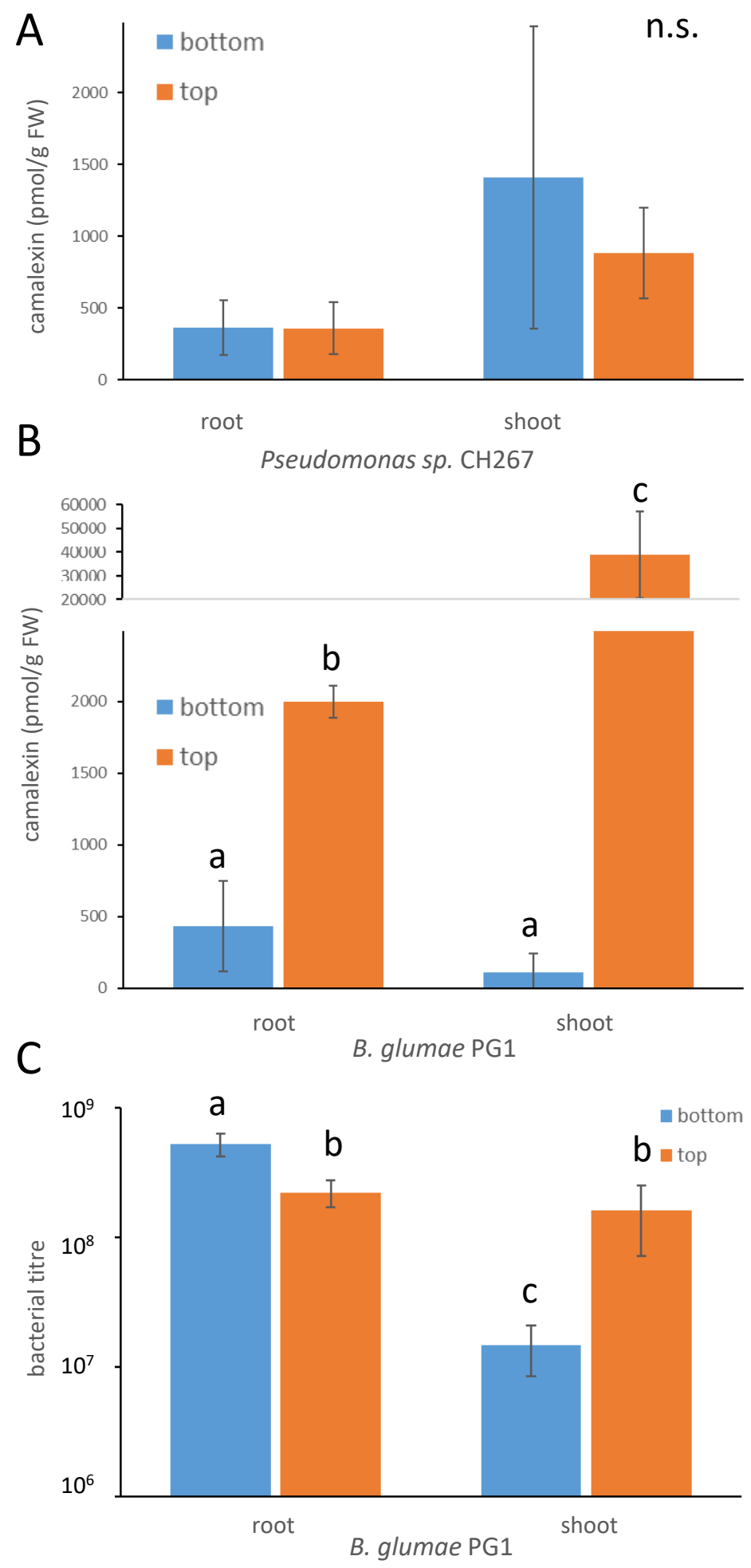

\section{Figure 3. Tissue specificity of camalexin synthesis}

Arabidopsis seedlings were grown for 14 days on an agar plate, inoculated with Pseudomonas sp. CH267 (A) or B. glumae PG1 (BG) (B) either on the leaves or on the root tips, and incubated for 3 days. Camalexin accumulation in leaves and roots was determined by HPLC. Data are presented as means \pm S.D. from 3 biological replicates, each corresponding to 3 individual roots or shoots. C DNA was isolated from the roots and shoots and subjected to qPCR with primers against B. glumae PG1 and Arabidopsis TIP41 gene as control. Using previously established calibration between Ct values, OD and cfu, the qPCR data were expressed as cfu, presented as means \pm S.D. from 4 biological replicates, each corresponding to 3 individual roots or shoots. Different letters mark values significantly different at $\mathrm{P}<0.05$ (T-test). 


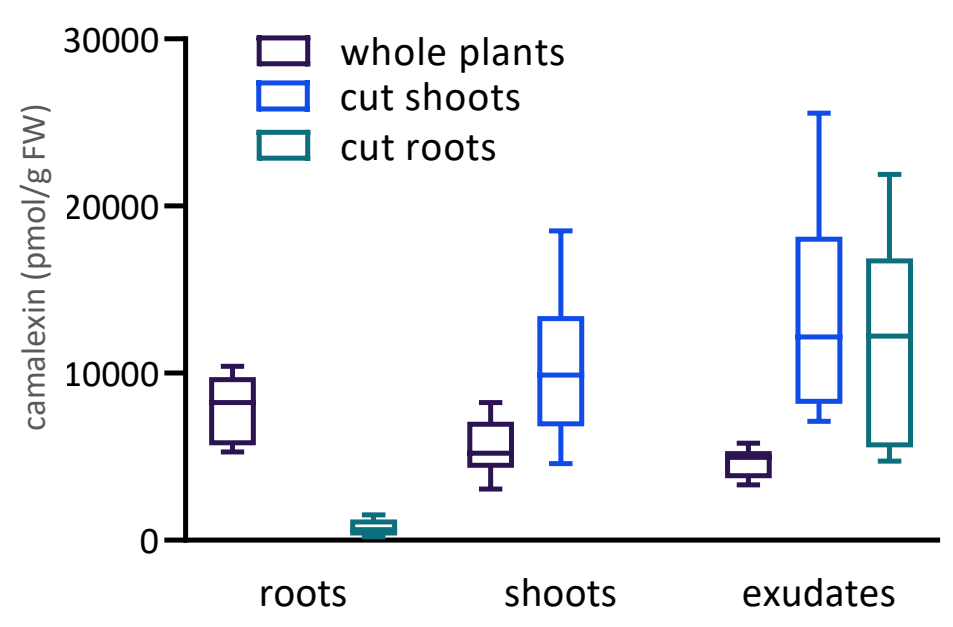

\section{Figure 4. Camalexin in cut roots and shoots}

Arabidopsis seedlings were grown on a nylon net in hydroculture for 10 days, the shoots were cut with scissors, the roots removed from the net and both placed separately to the solution. The shoots, roots, and intact plants were inoculated with B. glumae PG1 and further incubated for 3 days. Camalexin accumulation in shoots, roots, and exudates was determined by HPLC. Data are presented as box plots from at least 8 biological replicates, each corresponding to about 30 individual roots or shoots. The box extends from the $25^{\text {th }}$ to $75^{\text {th }}$ percentiles, the line is plotted at the median, the whiskers extend from minimum to maximum values. 


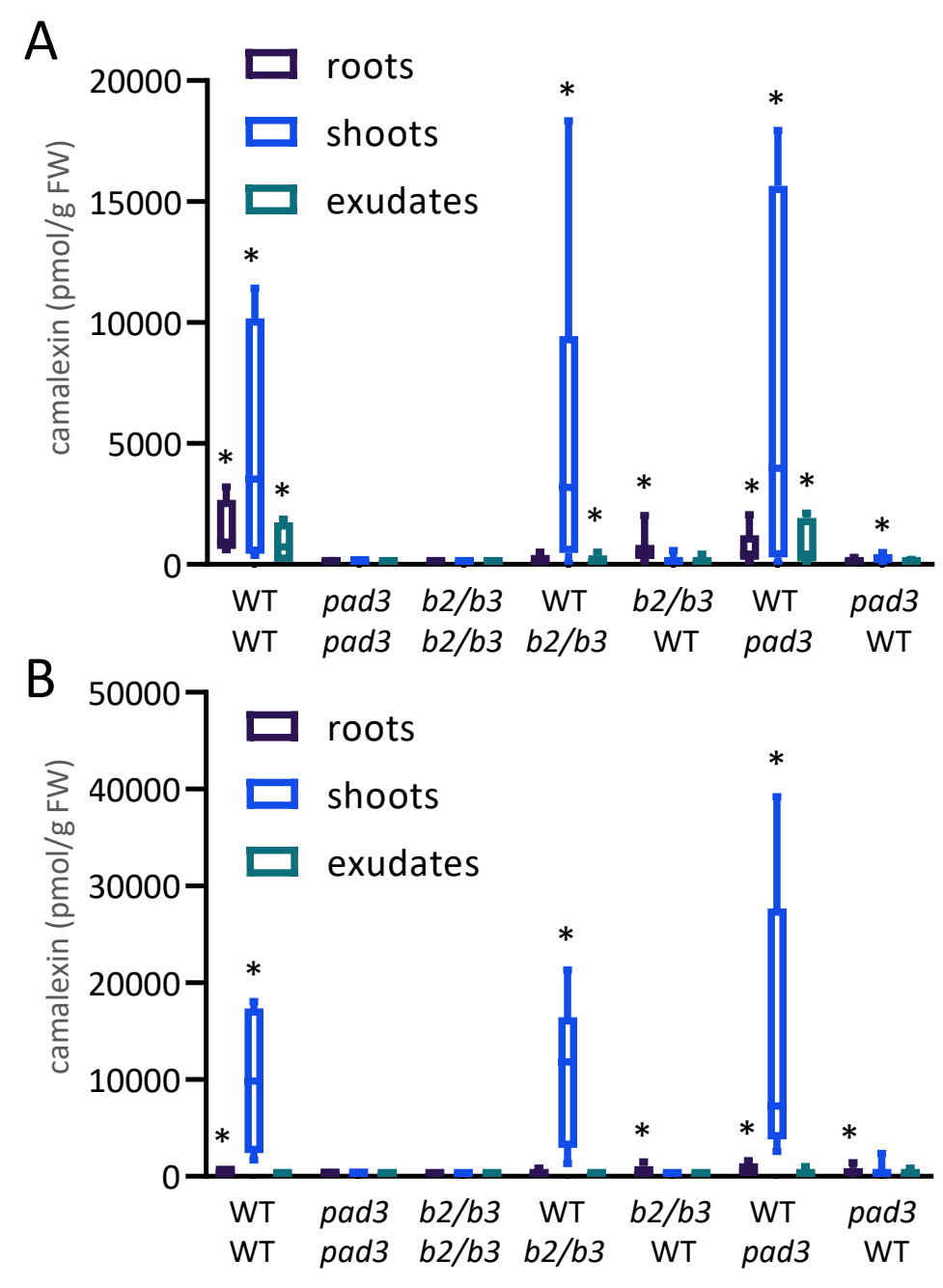

\section{Figure 5. Analysis of camalexin in grafted plants.}

Homografts of Arabidopsis WT, cyp79b2 cyp79b3 (b2/b3), and pad3 and the heterografts of the WT with the mutants were grown for 18 after the grafting, transferred onto cut caps of Eppendorf tubes and placed with only the roots submerged into the hydroculture solution. The plants were then inoculated with B. glumae PG1 into the solution (A) or onto the leaves (B) and further incubated for 3 days. Camalexin accumulation in shoots, roots, and exudates was determined by HPLC. Data are presented as box plots from at least 8 individual grafts. The box extends from the $25^{\text {th }}$ to $75^{\text {th }}$ percentiles, the line is plotted at the median, the whiskers extend from minimum to maximum values. 